1/320, speckled pattern, but no SSc-related autoantibody has been identified. He had gastrointestinal (upper gastrointestinal tract dysmotility), muscular (myositis) and cardiac (heart failure with secondary cardiac cirrhosis (LVEF 40\%, NYHA class III)) involvement, without major pulmonary involvement (no ILD or PAH). He underwent a cardiac transplant at the age of 54 , after a disease duration of 7 years. Standard immunosuppressants were initiated.

Case 3 is a 50 -year-old male with diffuse cutaneous SSc. ANA was negative. $\mathrm{He}$ had vascular (digital ulcers), gastrointestinal (upper gastrointestinal tract dysmotility) and cardiac (heart failure with secondary cardiac cirrhosis (LVEF $40 \%$, NYHA class III)) involvement, without major pulmonary involvement (no ILD or PAH). He underwent a cardiac transplant at the age of 49 , after a disease duration of 4 years. Standard immunosuppressants were initiated.

At present, 1,5 years (case 2 and 3 ) and 8 years (case 1) after transplant, the donor hearts are still functioning well. No other SSc-related organ manifestations have occurred.

Conclusions: We present 3 patients with SSc who successfully underwent cardiac transplant for SSc-related end-stage heart disease. None had other major SSc-related organ involvement. This supports the limited published data that cardiac transplant is feasible and can be considered in end-stage SSc-related cardiomyopathy.

Disclosure of Interest: None declared

DOI: 10.1136/annrheumdis-2017-eular.4251

\section{AB0639 SUPERIOR TREATMENT RESPONSE OF INTERSTITIAL LUNG DISEASE IN INFLAMMATORY MYOPATHIES COMPARED TO OTHER CONNECTIVE TISSUE DISEASES - A PROSPECTIVE COHORT STUDY}

J.J. Maya ${ }^{1}$, A. Borja ${ }^{2}$, A. Abril ${ }^{1}$, I. Mira-Avendaño ${ }^{2} .{ }^{1}$ Rheumatology;

${ }^{2}$ Pulmonary, Mayo Clinic Florida, Jacksonville, United States

Background: Interstitial Lung Disease (ILD) associated to Connective Tissue Disease (CTD) represent a challenge for clinicians and researchers because of their significant morbidity and mortality. Although the different types of ILD associated to CTD are often studied and managed as one because of their autoimmune background, there are considerable differences in their etiopathogenesis and therefore it can be assumed that there are differences in their response to treatment (1). Even though previous studies have analyzed the impact of immunosupipression in ILD secondary to sclerodema, additional studies are needed in order to determine the response to treatment of the different forms of ILD associated to CTD (2-3).

Objectives: To characterize and analyze the response to treatment of different types of ILD associated to CTD. The primary endpoint is the Functional Vital Capacity (FVC) change at 6 months and 1 year, and secondary endpoints arere the change in Diffusion Capacity of the Lung for Carbon Monoxide and in a 6 Minute Walk Test.

Methods: A prospective cohort study is being carried out where all patients who present to the Mayo Clinic Florida pulmonary clinic, age 18 to 80 , with established CTD and diagnosed with ILD, and all patients with ILD who meet the criteria for immunologic mediated process, are being followed for a year in order to evaluate the clinical and functional outcomes to treatment. Patients with moderate to severe Pulmonary Hypertension, and active smokers with bronchiolitis pattern are being excluded. Exploratory analysis were performed on the first group of patients enrolled in the study, continuous variables were described with central tendency measures and the mean absolute difference in adjusted 12-month FVC was analyzed between the different types of CTDs using student's t test.

Results: Thirteen patients with ILD were enrolled in the study's initial phase. Five of the patients had been diagnosed with an Inflammatory Myopathy (IM), 2 with Rheumatoid Arthritis, 1 with an Undifferentiated Connective Tissue Disease, 1 with Churg-Strauss Syndrome, and one with Systemic Sclerosis. One patient was treated with Rituximab only; 2 with Rituximab and a steroid; 3 with Mycophenolate Mofetil (MMF) only; 2 with steroids, MMF, and Rituximab; 1 with a TNF inhibitor and MMF; 1 with MMF and steroids; 1 with Azathioprine and steroids; and 1 received only steroids. IMs were compared to the rest. At 1 year follow-up FVC mean absolute difference for the IMs demonstrated and improvement of 0.43 while the other CTDs had worsen by a mean of $0.04(p=0.01)$. There were no statistical differences at 6 months for all outcomes or at 1 year for DLCO and 6MWT.

Conclusions: To our knowledge, this is the first time that a cohort of ILD associated to CTD patients is followed and analyzed after 1 year of treatment. Our results suggest that there is a difference in the response to treatment of ILD depending on the underlying CTD. Specific types of ILD associated to CTD likely benefit from early and aggressive treatment, but longer follow ups and larger studies are needed in order to be able to validate and further understand these pathologies.

References:

[1] Chest 2013; 143 (3): 814-824.

[2] Lancet Respir Med. 2016 Sep;4(9):708-19.

[3] Semin Arthritis Rheum. 2016 Oct 13.[Epub ahead of print]

Disclosure of Interest: None declared

DOI: 10.1136/annrheumdis-2017-eular.5653

\section{AB0640 DOES MIXED CONNECTIVE TISSUE DISEASE WITHOUT ANTI-U1RNP EXIST?}

J. Martínez-Barrio $^{1,2}$, E. Estrada ${ }^{3,4}$, J.G. Ovalles-Bonilla ${ }^{1}$, L. Valor ${ }^{1}$

D. Hernández-Flórez ${ }^{1}$, T. del Río ${ }^{1}$, I. Janta ${ }^{1}$, J.C. Nieto Gonzalez ${ }^{1}$, B. Serrano ${ }^{1}$, R. González Benítez ${ }^{1}$, C. Sáenz Tenorio ${ }^{1}$, L. García-Montoya ${ }^{1}$, M. Correyero ${ }^{1}$, A. Silva ${ }^{1}$, A. López-Cerón ${ }^{1}$, C. Gonzalez ${ }^{1}$, I. Monteagudo ${ }^{1}$, F.J. López-Longo ${ }^{1}$. ${ }^{1}$ Department of Rheumatology, Hospital General Universitario Gregorio

Marañón; ${ }^{2}$ Universidad Complutense de Madrid; ${ }^{3}$ Facultad de Salud, Universidad Camilo José Cela; ${ }^{4}$ Facultad de Psicología, Universidad Autónoma de Madrid, Madrid, Spain

Background: Mixed Connective Tissue Disease (MCTD) is a systemic autoimmune rheumatic disease (SARD) characterized by clinical manifestations of systemic lupus erythematosus (SLE), systemic sclerosis (SSc) and polymyositis (PM) and the presence of anti-U1-RNP antibodies.

Objectives: To determine whether there are patients with symptoms of MCTD in the absence of anti-U1-RNP antibodies.

Methods: This was a monocentric, prospective, observational study of patients with SARD. All patients diagnosed of MCTD according to Kasukawa and/or Alarcón-Segovia's criteria, SLE, SSc, PM, overlap syndromes (simultaneous or sequential criteria of 2 or more SARD), Sjögren's syndrome, Antiphospholipid syndrome, systemic vasculitis and undifferentiated or incomplete SARD (at least one clinical criterion of the classification criteria and a related antibody of any of the SARD) were included in the "Autoimmune Systemic Rheumatic Diseases Registry" of the Hospital General Universitario Gregorio Marañon Rheumatology Department from 1986 to 2012. The registry includes 2406 patients diagnosed with SARD. Patients with rheumatoid arthritis were excluded. Patients with clinical MCTD criteria were divided into seropositive (MCTD, with anti-U1RNP) and seronegative (possible MCTD, without anti-U1RNP). The registry counts with the local Institutional Ethics Board approval.

Results: A total of 692 patients were recruited, 608 women (87.9\%). Seventy $(70$, $10.1 \%$ ) patients were classified as seropositive and $75(10.8 \%)$ as seronegative by Kasukawa's criteria. Sixty-two $(62,8.9 \%)$ patients were classified as seropositive and $54(7.8 \%)$ as seronegative according to Alarcón-Segovia's criteria. There were no significant differences in age at disease onset, age at diagnosis or disease duration $(p>0.05)$ between seropositive and seronegative patients. Seropositive patients with Kasukawa's criteria presented more frequently: lymphadenopathy, malar rash, leukopenia, Raynaud's phenomenon, muscle weakness and increase of muscle enzymes (Table 1). By Alarcón-Segovia's criteria, patients who developed myositis were more frequent in the seropositive group $(p=0.007, O R$ $3.25,95 \% \mathrm{Cl}, 1.44-7.32)$.

\begin{tabular}{|c|c|c|c|c|c|c|c|c|}
\hline \multirow[t]{2}{*}{ Kasukawa criteria } & \multicolumn{2}{|c|}{$\begin{array}{l}\text { Seropositive } \\
\text { MCTD }\end{array}$} & \multicolumn{2}{|c|}{$\begin{array}{c}\text { Seronegative } \\
\text { MCTD }\end{array}$} & \multirow[t]{2}{*}{$P$} & \multirow[t]{2}{*}{ OR } & \multicolumn{2}{|c|}{$\begin{array}{c}95 \% \mathrm{Cl} \\
\mathrm{OR} \\
\end{array}$} \\
\hline & $\bar{n}$ & $\%$ & $N$ & $\%$ & & & $\operatorname{lnf}$ & Sup \\
\hline & 28 & $40 \%$ & 17 & $23 \%$ & 0.038 & 2.275 & 1.105 & 4.681 \\
\hline Mals & 25 & $36 \%$ & 8 & $11 \%$ & 0.001 & 4.653 & 1.928 & 11.231 \\
\hline Leucopenia & 41 & $59 \%$ & 17 & $23 \%$ & $<0.001$ & 4.824 & 2.348 & 9.909 \\
\hline Muscle weakness & 32 & $50 \%$ & 24 & $32 \%$ & 0.042 & 2.125 & 1.083 & 4.171 \\
\hline Increase of muscle enzymes & 45 & $64 \%$ & 35 & $47 \%$ & 0.049 & 2.057 & 1.056 & 4.008 \\
\hline
\end{tabular}

Conclusions: Some patients with SARD manifestations fulfill MCTD clinical criteria, both Kasukawa's and Alarcón-Segovia's, in the absence of anti-U1-RNP antibodies from the onset of the disease and throughout its evolution (seronegative MCTD). The frequency of seronegative MCTD was similar to the frequency of seropositive MCTD. Patients with seropositive MCTD presented more frequently manifestations of SLE (lymphadenopathy, malar rash and leukopenia) when using Kasukawa's criteria and of PM when using both criteria.

Disclosure of Interest: None declared

DOI: 10.1136/annrheumdis-2017-eular.5192

\section{AB0641 ARTERIAL STIFFNESS AND CLINICAL ASSOCIATION IN PATIENTS WITH SYSTEMIC SCLEROSIS}

K. De La Cruz Rodríguez ${ }^{1}$, G. Martínez Bonilla ${ }^{2}$, V. González Díaz ${ }^{2}$, K. García Osuna $^{2}$, J. Reyes Rueda ${ }^{2}$, C. Gómez López ${ }^{2}$, J. Polanco Cruz ${ }^{2}$, A. Bernard Medina $^{2}$, E. Cardona ${ }^{3}$, A. Macías Chumacera ${ }^{4}$, C. Ramos Becerra ${ }^{4}$,

S. Gutiérrez Ureña ${ }^{2}$, S. Cerpa Cruz ${ }^{2} \cdot{ }^{1}$ Rheumatology Service; ${ }^{2}$ Hospital Civil Fray Antonio Alcalde; ${ }^{3}$ Mechanical Vascular Service; ${ }^{4}$ CUCS, Guadalajara, Jalisco, Mexico

Background: Systemic sclerosis is an autoimmune disease characterized by microvascular damage and fibrosis. There are several studies that shown macrovascular damage with arterial stiffness (AS) and the risk of cardiovascular complications. Carotid-femoral pulse wave velocity (CF-PWV) and augmentation index $(A \mid x)$ are two competent methods to determine AS and predictors of cardiovascular disease. Association between AS and microvascular damage is unknown in systemic sclerosis patients.

Objectives: To determine the frequency of arterial stiffness in patients with systemic sclerosis and its association with clinical manifestations.

Methods: We performed a cross-sectional study; patients with diagnosis of systemic sclerosis according to ACR/EULAR 2013 criteria were included and the control group was selected from a database of mechanical vascular service. AS 
was non-invasively assessed by Pulse pen device and Alx was evaluated by tonometry. Statistical analysis was done with SPSS v22 software, we calculated mean and standard deviation, for continuous variables we used Student $t$ test, categorical variables were analyzed by using chi-square or Fisher's exact test. The correlation of AS and clinical variables was assessed with Spearman's correlation.

Results: Forty seven patients were included and compared with 39 healthy subjects; mean age of study group was $48 \pm 14$. vs control group $47 \pm 13.7 \quad(p=0.08)$ $93 \%$ were female. Prevalence of AS was $11 \%$ vs $3 \% p=0.039$. AS was more frequent in limited systemic sclerosis sub-group and we found correlation with abnormal capillaroscopy, Rho $0.292 \mathrm{p}=0.04$.

Conclusions: Arterial stiffness is more prevalent in patients with limited systemic sclerosis and association with abnormal caillaroscopy suggest that both macro and microvascular damage is present in these patients and could explain the presence of early atherosclerosis and increased risk of cardiovascular disease.

References:

[1] Vargas John, Lafyatis Robert. Etiology and pathogenesis of systemic sclerosis. Marc Hochberg. Texbook of Rheumatology (Sixth Edit). Elsevier, 2015. 11771245.

[2] Ngian G.-S., Sahhar J., Wicks I., Van Doornum, S. Arterial stiffness is increased in systemic sclerosis: A cross-sectional comparison with matched controls. Arthritis and Rheumatism 2012. 64, S301.

[3] Colaci M., Giuggioli D., Manfredi A., Sebastiani M. Aortic pulse wave velocity measurement in systemic sclerosis patients, Rheumatism 2012. 64(6), 360367.

[4] Man A., Zhu Y., Zhang Y., Dubreuil M., Rho Y. H., Peloquin C. Choi, H. K. The risk of cardiovascular disease in systemic sclerosis: a population-based cohort study. Annals of the Rheumatic Diseases 2013. 72(7), 1188-93.

Disclosure of Interest: None declared

DOI: 10.1136/annrheumdis-2017-eular.3345

\section{AB0642 THICKNESS OF THE INTIMA-MEDIA COMPLEX AND DOPPLER VELOCITY PARAMETERS IN SCLERODERMA PATIENTS}

K. Rutka ${ }^{1}$, E. Gindzienska-Sieskiewicz ${ }^{2}$, R. Milewski ${ }^{3}$, S. Sierakowski ${ }^{2}$ U. Lebkowska ${ }^{1} .{ }^{1}$ Radiology; ${ }^{2}$ Rheumatology; ${ }^{3}$ Statistics and Medical Informatics, Medical University of Bialystok, Bialystok, Poland

Background: Systemic sclerosis (Ssc) is a chronic inflammatory autoimmune disease, that involves various tissues and organs, including the cardiovascular system. Ssc patients might suffer from a number of complications - including cardiovascular system diseases. Diagnostic imaging is an important tool in assessment of vascular lesions, and ultrasound (including Doppler ultrasound) is one of the most important examinations, which allow to scan for presence of atherotic lesions, assess the intima-media complex thickness as well as provide measurements of the blood flow parameters.

Objectives: The aim of study was to determine if there is any difference in the intima-media complex thickness as well as in blood flow parameters measured using Doppler ultrasound examination in scleroderma patients and the general population.

Methods: 35 patients, aged 19-75, with diagnosed systemic scleroderma were examined using a Doppler ultrasound examination. Thickness of intima-media complex (IMT) approximately 2 centimeters from the carotid bulb was assessed for both right and left common carotid artery (CCA). The standard parameters of blood flow were measured - including peak systolic velocity (PSV), end diastolic velocity (EDV), as well as resistive index (RI), pulsative index (PI) and standard deviation (SD) was measured in the common carotid arteries, internal carotid arteries (ICA) as well as in the vertebral arteries (VA).

Results: The mean IMT value in CCA was approximately $0.63 \mathrm{~mm}(0.35-$ $0.9 \mathrm{~mm})$.

The mean, minimal and maximal values measured in CCA equalled respectively: PSV - $68.7 \mathrm{~cm} / \mathrm{s}(22.4-94 \mathrm{~cm} / \mathrm{s})$, EDV - 19,7 cm/s $(4.7-30.35 \mathrm{~cm} / \mathrm{s}), \mathrm{PI}-$ $1.95(0.41-2,09)$ and $\mathrm{RI}-0.68(0.28-0.81)$. In ICA the measured values were as follows: PSV $-68.4 \mathrm{~cm} / \mathrm{s}(38.65-96.9 \mathrm{~cm} / \mathrm{s})$; EDV $-23.6 \mathrm{~cm} / \mathrm{s}(9.05-38.6$ $\mathrm{cm} / \mathrm{s}), \mathrm{PI}-1.16(0.76-1.71)$ and $\mathrm{RI}-0.65(0.37-0.79)$; in VA PSV $-46.5 \mathrm{~cm} / \mathrm{s}$ $(26.75-75.35 \mathrm{~cm} / \mathrm{s}) ; \mathrm{EDV}-12.4 \mathrm{~cm} / \mathrm{s}(6.1-22 \mathrm{~cm} / \mathrm{s}), \mathrm{PI}-1.38(0.6-2.28)$ and $\mathrm{RI}$ $-0.73(0.34-1.33)$.

A positive correlation between the age of examined subjects and the IMT was found $(p=0.002 ; R=0.49$ ). Additionaly, a negative correlation between the IMT and the EDV was found $(p=0.008, R=-0.44)$.

Conclusions: The mean thickness of intima-media complex in the examined group of SSc patients is within the values that were established for a healthy population, however a further investigation, including a control group study will allow to evaluate whether there is no correlation between systemic sclerosis and increased IMT.

A negative correlation between IMT and EDV was shown, which is an interesting finding, and could be confirmed in a control group study and on a larger group of patiens.

References:

[1] Kochanowicz J. et al Normal reference values of ratios of blood flow velocities in internal carotid artery to those in common carotid artery using Doppler sonography. J Clin Ultrasound. 2009 May;37(4):208-11.

[2] Buljan K et al. Relationship between Age and Thickness of Carotid Arteries in a Population without Risk Factors for Atherosclerosis. Coll Antropol. 2015 Sep;39(3):779-84.

[3] Yazici B. et al. Cerebral blood flow measurements of the extracranial carotid and vertebral arteries with Doppler ultrasonography in healthy adults. Diagn Interv Radiol. 2005 Dec;11(4):195-8.

Disclosure of Interest: None declared

DOI: 10.1136/annrheumdis-2017-eular.6296

\section{AB0643 MALIGNANCY SCREENING IN AUTOIMMUNE MYOSITIS AMONGST AUSTRALIAN RHEUMATOLOGISTS}

K. Dutton ${ }^{1}$, M. Soden ${ }^{2} .{ }^{1}$ Rheumatology, RBWH, Brisbane; ${ }^{2}$ Rheumatology, Townsville Hospital, Douglas, Australia

Background: The association between cancer and autoimmune myositis is well established and has lead to the common practice of malignancy screening in asymptomatic individuals. The international literature advocates widely for cancer screening in autoimmune myositis however no consensus or quideline has been published to set forth a process for screening standardisation (1). Malignancy screening is a complicated topic and recommendations in favour of screening should in principle be based on a judicious assessment of the evidence in terms of the benefits, risks and costs. In inflammatory myositis there is currently insufficient evidence to support any recommendation with respect to cancer screening. In the absence of clinical guideline and quality evidence, our study aimed to establish the current trends in malignancy screening amongst Australian Rheumatologists. Objectives: To explore the current trends in malignancy screening in autoimmune myositis amongst Australian Rheumatologists using an online questionnaire. Methods: Research approval was granted by The Townsville Hospital. An invitation email containing the survey weblink was sent twice to 386 Australian Rheumatologists between August 2015 and August 2016. Voluntary participation and anonymity were guaranteed. The questionnaire contained a fixed set of multiple choice questions that requested data on respondent demographics, practice setting and screening preference, practice and concerns. Open entry comment was an option throughout the questionnaire. Fifty-eight Rheumatologists, 1 Immunologist and 1 Paediatric Rheumatologist responded (16\% response rate). There were 3 survey dropouts. The data was pooled, coded and analysed using statistical software. All data was included in the analysis.

Results: Most respondents $(\mathrm{N}=58)$ were in private $(67 \%)$ and/or public practice $(68 \%)$, in practice for $>10$ years $(70 \%)$, conducted cancer screening $(93 \%)$ and were "very" or "somewhat" confident in their screening practice $(90 \%)$. The majority $(72 \%)$ performed cancer screening independent of patient characteristics. Determinants that triggered screening (in descending order of popularity) were: tobacco use $(\mathrm{N}=11)$, history of cancer $(\mathrm{N}=10)$, age $>40(\mathrm{~N}=7)$, cancer family history $(\mathrm{N}=7)$, age $>50(\mathrm{~N}=3)$ and age $>60(\mathrm{~N}=1)$. Most respondents indicated preference to order screening tests (in descending order of popularity): mammogram $(81 \%)$; CT chest \& abdomen (78\%); myeloma screen $(70 \%)$; chest x-ray $(69 \%)$, serum PSA $(67 \%)$, PAP smear $(54 \%)$, colonoscope $(44 \%)$, LDH (41\%), pelvic USS $(33 \%)$, gastroscope $(33 \%)$, FOBT $(33 \%)$, tumour markers $(28 \%)$, CT neck $(17 \%)$, nuclear bone scan $(15 \%)$, PET CT $(4 \%)$ \& testicular USS $(2 \%)$. Respondents $(\mathrm{N}=57)$ indicated that cancer screening was problematic due to a lack of clinical practice consensus \& guideline $(77 \%)$, test selection knowledge $(37 \%)$ and knowledge regarding repeated screening (53\%). The potential for harm in conducting screening was identified to be a problem by most respondents $(62 \%)$. Conclusions: The practice of malignancy screening in autoimmune myositis amongst Australian Rheumatologists is highly variable. Practice is driven by patient factors and clinician preferences. The cancer screening process is felt on several fronts to have inherent problems. Guideline, consensus and further research is needed in this area to address the challenges and evidence gap.

References:

[1] Masiak, A et al. (2016). Clinical characteristics of patients with anti-TIF1-y Abs. Reumatologia. 54(1): 14-18.

Disclosure of Interest: None declared

DOI: 10.1136/annrheumdis-2017-eular.1148

\section{AB0644 FINGERPRINT ABNORMALITIES IN SYSTEMIC SCLEROSIS: A SINGLE CENTER SURVEY FROM INDIA}

K. Chanakya, P.K. Devarasetti, R.V.P. Irlapati, L. Rajasekhar. Rheumatology, Nizams institute of medical sciences, Hyderabad, India

Background: Fingerprint [FP] abnormalities are known in patients with Systemic Sclerosis [SSc]. Little has been described about their frequency, systemic associations and social impact in literature.

Objectives: To study the fingerprint abnormalities in Systemic Sclerosis patients Methods: Raynaud's phenomenon [RP] was taken as the inclusion criteria. Patients with SSc [limited LcSSc and diffuse DcSSc], SSc overlap with other Connective Tissue Diseases [CTDs] and other CTDs with RP were screened for FP abnormalities using a Standardization Testing and Quality Certification (STQC) Directorate certified biometric FP scanner. FP quality assessment was done by recording The National Institute of Standards and Technology [NIST] finger print image quality [NFIQ] scores ${ }^{1}$. NFIQ's 5 levels of quality are intended to be predictive of fingerprint matching. $\mathrm{NFIQ}=1$ indicates high quality samples and $\mathrm{NFIQ}=5$ indicates poor quality samples. Other associated systemic features of 